\title{
Illuminating the impact of sub-micron particle size and surface chemistry on interfacial position and Pickering emulsion type.
}

\section{- Supporting Information -}

Emma C. Giakoumatos ${ }^{\ddagger a, b, d}$, Antonio Aloi ${ }^{\ddagger a, c, d}$, and Ilja K. Voets ${ }^{* a, c, d}$

aLaboratory of Self-Organizing Soft Matter, 'baboratory of Physical-Chemistry, 'Laboratory of Macromolecular and Organic Chemistry and Institute for Complex Molecular Systems, Eindhoven University of Technology

${ }^{\ddagger}$ Equal contribution

*Corresponding author: I.Voets@tue.nl 


\section{Contents}

1. Materials and methods

1.1 Pickering emulsion preparation

1.2 Confocal microscopy

1.3 iPAINT probe synthesis

1.4 iPAINT sample preparation

1.5 iPAINT super-resolution microscopy.

1.6 iPAINT image analysis.

2. Particle characterization

2.1 SEM Carboxyl polystyrene latex colloids

2.2 SEM Silica colloids

$2.3 \zeta$-potential measurements

3. Silica colloids adsorbed at water-octanol interfaces

4. Extended contact angle data.

4.1 $\theta$ vs. diameter for carboxyl-polystyrene and silica particles.

$4.2 \theta$ vs. diameter for carboxyl-polystyrene particles at different $\mathrm{pH}$.

5. Contact angle dependency on $\mathrm{pH}$.

6. References. 


\section{Materials and methods.}

\subsection{Pickering emulsions preparation.}

2-Octanol was purchased from Fluka Chemical and used without further treatment to formulate Pickering emulsions. Silica (Micromod) and carboxyl polystyrene particles (Spherotech) were washed 10 times to replace the storing buffer, containing surfactants preventing the particle aggregation, with milli-Q water $(18.2 \mathrm{M} \Omega)$. Once washed the particles were dispersed in water at the desired concentration, the $\mathrm{pH}$ of which was adjusted using small amounts of $\mathrm{NaOH}$ or $\mathrm{HCl}$. All CPS particles have the same surface charge density of 1.33 groups $/ \mathrm{nm}^{2}$. The oil phase in was stained with Nile red solvatochromic dye (Sigma Aldrich) to distinguish continuous and dispersed phases. Pickering emulsions were produced by mixing $1 \mathrm{ml}$ of particle dispersion ( $2 \mathrm{wt} \%$ ) with $1 \mathrm{ml}$ of octanol ( $0.05 \mathrm{wt} \%$ Nile red) using a T25 homogenizer at 13000 rpm for 2 minutes.

\subsection{Confocal microscopy.}

After emulsification $20 \mu \mathrm{L}$ of the Pickering emulsion was placed between a coverslide (Menzel Gläser, $76 \times 26 \mathrm{~mm}$, thickness $1 \mathrm{~mm}$ ) and a coverslip (Menzel Gläser, No. 1.5, $24 \times 24 \mathrm{~mm}$, thickness $170 \mu \mathrm{m}$ ) using an imaging spacer (Grace Bio-Labs SecureSeal ${ }^{\mathrm{TM}}, 1$ well, diameter $x$ thickness $13 \mathrm{~mm} \times 0.12 \mathrm{~mm}$ ). All confocal images were acquired on a Leica TCS-SP8 inverted microscope using a 10x or 20x dry objective.

\section{3 iPAINT probes synthesis.}

The synthesis of iPAINT probes is described elsewhere. ${ }^{15}$ Briefly, $\sim 1 \mathrm{mg}$ of poly(ethylene glycol) (PEG) bis(amine) (MW 20kDa, Sigma Aldrich) was set to react with $20 \mu \mathrm{L}$ of $\mathrm{N}$-hydroxysuccinimide ester activated rhodamine $10 \mathrm{mM}$ (Cage552, Abberior ${ }^{\circledR}$ ) in $1 \mathrm{~mL}$ of $0.1 \mathrm{M}$ sodium bicarbonate buffer at $\mathrm{pH}$ 8.5. The reaction mixture was protected from light and stirred overnight at room temperature. Subsequently, the product (PEG-552) was purified by dialysis (Spectra/Por ${ }^{\circledR} 7$ dialysis membrane, pretreated RC tubing, molecular weight cutoff: $8 \mathrm{kDa}$ ) to remove unreacted dyes.

\section{4 iPAINT sample preparation.}

Stock solutions of carboxyl polystyrene latex particles $(0.002 \% \mathrm{w} / \mathrm{v})$ were dispersed in a $10^{-5} \mathrm{M}$ aqueous solution of the iPAINT dye PEG-552. $15 \mu \mathrm{L}$ of this solution was placed onto a coverglass (Menzel Gläser, $76 \times 26 \mathrm{~mm}$, thickness $1 \mathrm{~mm}$ ) next to a $15 \mu \mathrm{L}$ droplet of 2-octanol. The sample was sandwiched between the coverslide (Menzel Gläser, $76 \times 26 \mathrm{~mm}$, thickness $1 \mathrm{~mm}$ ) and a coverslip (Menzel Gläser, No. 1.5, $24 \times 24 \mathrm{~mm}$, thickness $170 \mu \mathrm{m}$ ) using double-sided tape (thickness $\sim 90 \mu \mathrm{m}$ ) (Fig. S1). The coverslips were treated with piranha etching (piranha solution: 3:1 v/v concentrated $\mathrm{H}_{2} \mathrm{SO}_{4}: \mathrm{H}_{2} \mathrm{O}_{2}$ (aq. $\left.\left.30 \%\right)\right)$ to remove impurities and reduce background fluorescence. No solvent evaporation was observed during the image acquisition procedure thanks to fast imaging ( $\sim 20 \mathrm{~min}$ for a $256 \times 256$ pixel field of view). 


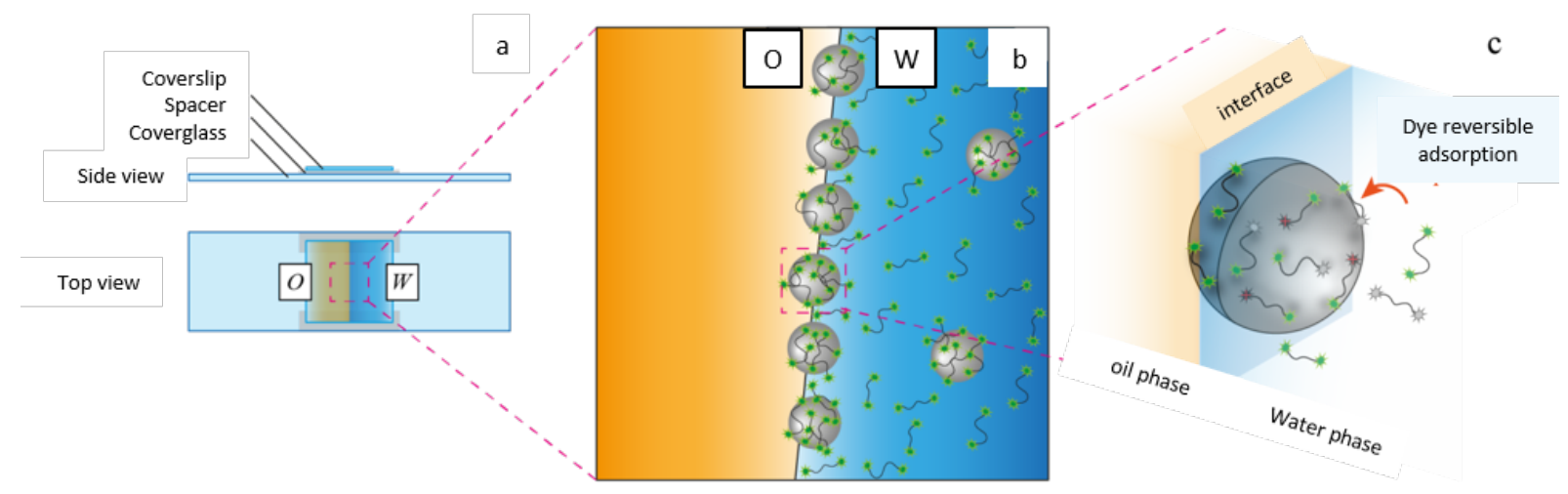

Fig. S1: Schematic illustration of a fluid cell for iPAINT imaging of particles at a fluid interface. (a) Side and top view of the assembled chamber made out of a coverglass, double-sided tape and a coverslip. The two liquids are put into contact to create an interfacial plane normal to the coverglass. (b) Cartoon depicting colloidal particles adsorbed at the fluid interface between the water phase, rich in PEG-552 (green probes), and the oil phase. (c) Zoom-in on a particle adsorbed at the fluid interface in 3D.

\section{5 iPAINT super-resolution microscopy.}

iPAINT images were acquired on a Nikon Eclipse Ti-E N-STORM inverted microscope supplied with $\sim 488.0 \mathrm{~mW} / \mathrm{cm}^{2}(\lambda=561 \mathrm{~nm})$ and $\sim 160.0 \mathrm{~mW} / \mathrm{cm}^{2}(\lambda=405 \mathrm{~nm})$ laser lines set for quasi-TIRF imaging. The TIRF angle is adjusted to maximize the signal-to-noise ratio. A 100x, 1.49 NA oil immersion objective is used to collect the fluorescence signal which is subsequently filtered by a quad-band pass dichroic mirror (97335 Nikon). iPAINT images were acquired on an ixon3, Andor EMCCD camera (pixel size $17 \mu \mathrm{m}$ ) at a frame rate of 47 frames/s for a region of interest of $256 \times 256$ pixels. $5 \times 10^{4}$ frames are collected for each super-resolved image, during which the adsorption of the iPAINT probes at the fluid interfaces is observed. The PEG-552 probes are continuously photo-activated with a low power UV laser ( $405 \mathrm{~nm}, 0.5 \%$ power), excited and subsequently bleached using a $561 \mathrm{~nm}$ laser (100\% power). Single-molecule localization movies are analysed with NIS-element Nikon software.

\section{6 iPAINT image analysis.}

The data analysis of iPAINT images of particles straddling fluid interfaces is reported elsewhere. ${ }^{1}$ Briefly, being dispersed in the iPAINT probe solution, the particles are fully labelled with PEG-552. We isolate the particles using density filters applied to the number of single-molecule localization and proceed to fit the particles with a Matlab routine ('Circle Fit'). Here, the algorithm minimizes the quantity: $\sum\left(x^{2}+y^{2}-R^{2}\right)^{2}$. The fit gives $(x, y)$ coordinates of the centre of the particle and its radius. The position of the particle respect to the interface is determined identifying the single molecule localizations belonging to the interface and tracing its position across the field of view. ${ }^{1}$ By overlapping the profile of the interface with the position of the particles we can calculate the height of the hydrophilic particles $(h)$ in the oil phase and the lateral dimension of this hemisphere. Knowing the size of the particle, the height and the lateral size of the hemisphere, we can compute the contact angle, through the aqueous phase $\theta$ by trigonometry: 


$$
\theta=\cos ^{-1}\left(\frac{R-h}{R}\right)
$$

\section{Particle characterization.}

A FEI Quanta 600F ESEM scanning electron microscope was used to image the colloidal particles.

\subsection{SEM Carboxyl polystyrene latex colloids.}

Carboxyl polystyrene latex particles $(\varnothing=320 \mathrm{~nm}, 450 \mathrm{~nm}, 510 \mathrm{~nm}$ and $810 \mathrm{~nm}$ ) were purchased from Spherotech. All particles were washed 10 times to replace the storing buffer, containing surfactants preventing the particle aggregation, with milli-Q water (18.2 M $)$ ). Fig. S2 shows SEM images of carboxyl polystyrene latex particles (top row), with relative size distributions (bottom row) obtained from image analysis of more than 100 particles. The image analysis was completed using Image-J. From SEM image analysis we obtained mean diameters of $336 \pm 28 \mathrm{~nm}, 426 \pm 17 \mathrm{~nm}, 453 \pm 17 \mathrm{~nm}$, and 806 $\pm 28 \mathrm{~nm}$, while iPAINT imaging gave mean diameters of $298 \pm 51 \mathrm{~nm}, 435 \pm 16 \mathrm{~nm}, 515 \pm 31 \mathrm{~nm}$, and $809 \pm 39 \mathrm{~nm}$.

A
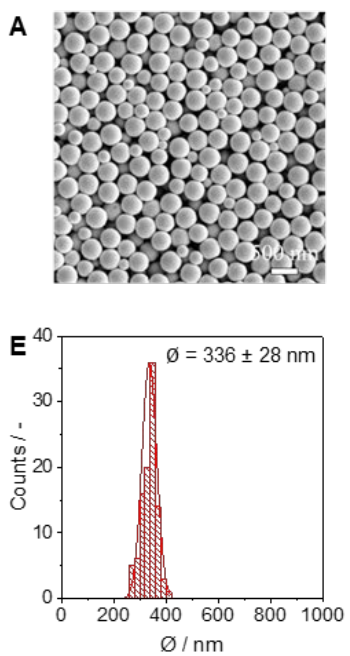

B
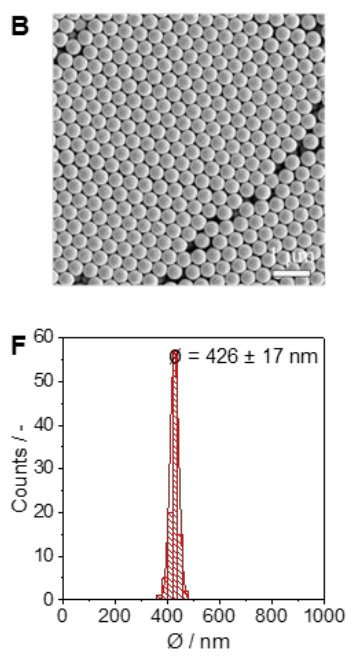
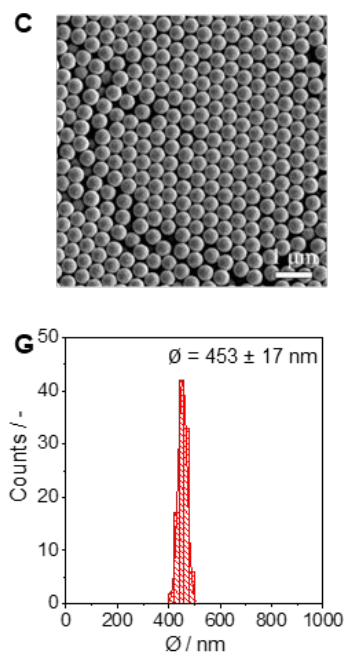

D
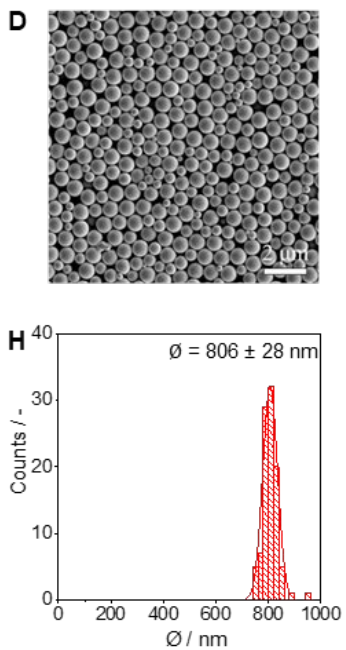

Fig. S2: (a-d) SEM images of carboxyl polystyrene latex particles. (e-h) size distribution of particle diameters measured for $n$ $>100$ particles. (e-h) size distribution histograms of single batches of carboxyl polystyrene latex particles. 


\subsection{SEM Silica colloids.}

Silica particles $(\varnothing=100 \mathrm{~nm}, 300 \mathrm{~nm}, 500 \mathrm{~nm}$ and $700 \mathrm{~nm}$ ) were purchased from Micromod. All particles were washed 10 times to replace the storing buffer, containing surfactants preventing particle aggregation, with milli-Q water (18.2 M $\Omega$ ). Fig. S3 illustrates the SEM images of silica particles (Fig. S3ad) and their size distributions (Fig. S3e-h) obtained from image analysis of more than 100 particles. The image analysis was completed using Image-J. From SEM image analysis we obtained mean diameters of $84 \pm 14 \mathrm{~nm}, 310 \pm 49 \mathrm{~nm}, 513 \pm 29 \mathrm{~nm}$, and $702 \pm 30 \mathrm{~nm}$, while iPAINT imaging gave mean diameters of $77 \pm 19 \mathrm{~nm}, 296 \pm 55 \mathrm{~nm}, 501 \pm 41 \mathrm{~nm}$, and $684 \pm 32 \mathrm{~nm}$.

A
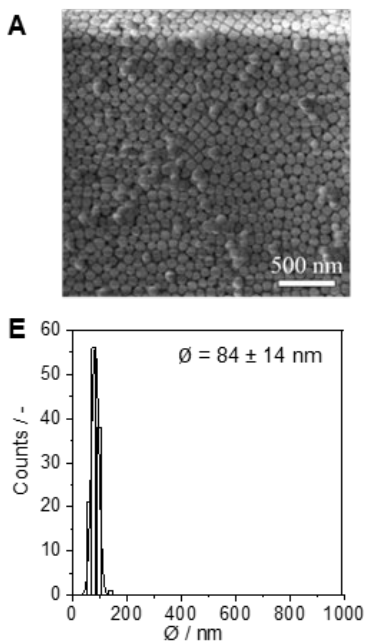

B
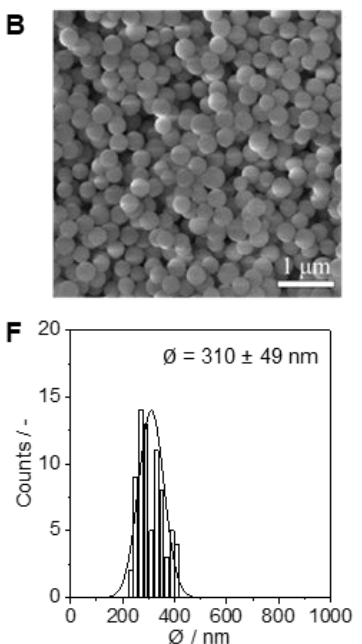

C
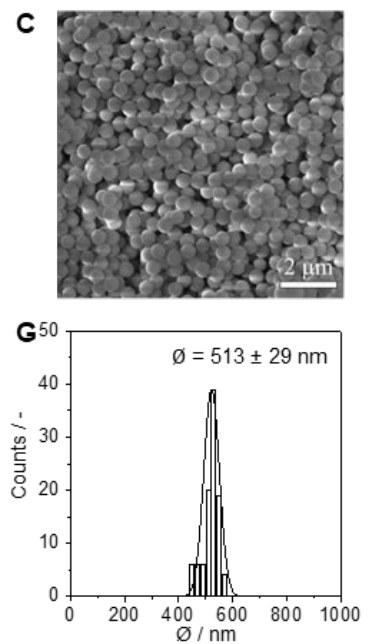

D
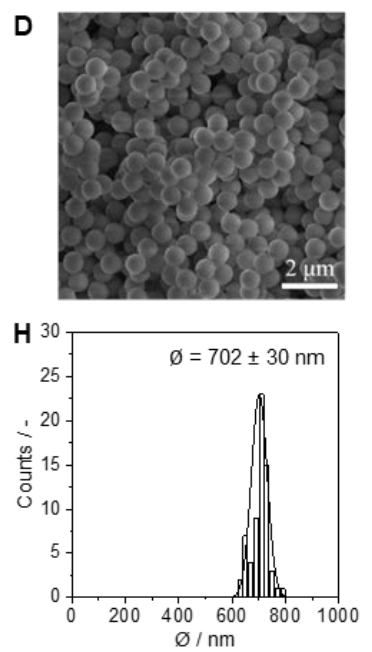

Fig. S3: (a-d) SEM images of silica particles. (e-h) size distribution of particle diameters measured for $n>100$ particles. (e-h) size distribution histograms of single batches of silica particles. 


\section{$2.3 \zeta$-potential measurements.}

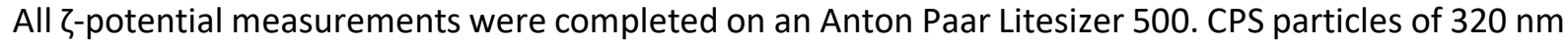
and $810 \mathrm{~nm}$ diameter were dispersed at $0.002 \mathrm{wt} \%$ in $1 \mathrm{mM} \mathrm{KCl}$, the $\mathrm{pH}$ of which was adjusted using small amounts of $\mathrm{HCl}$ and $\mathrm{NaOH}$.

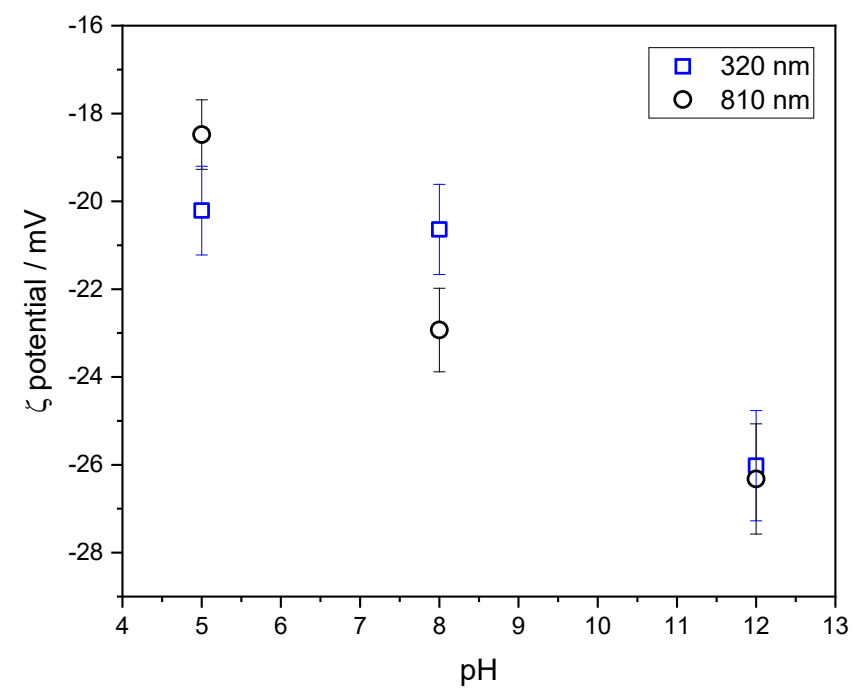

Fig. S4: $\zeta$-potential values for $320 \mathrm{~nm}$ and $810 \mathrm{~nm}$ CPS particles at different $\mathrm{pH}$ values. A clear trend of increasing charge in relation to $\mathrm{pH}$ is visible.

Measurements of CPS particles in the presence of the iPAINT probe were prepared with $0.002 \mathrm{wt} \%$ CPS particles, in a $1 \mathrm{mM} \mathrm{KCl}$ solution enriched with PEG-552 at $10^{-5} \mathrm{M}$.

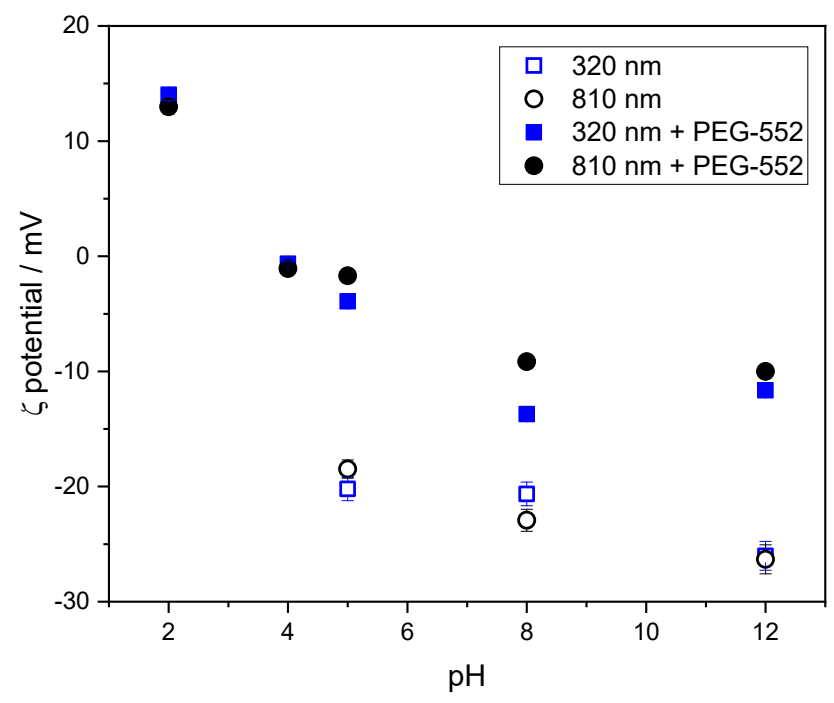

Fig. S5: క-potential values for $320 \mathrm{~nm}$ and $810 \mathrm{~nm}$ CPS particles at different pH values, with and without PEG-552 iPAINT probe. A clear trend of increasing charge in relation to $\mathrm{pH}$ is visible. 


\section{Silica colloids adsorbed at water-octanol interfaces.}

Silica particles of 100, 300, 500 and $700 \mathrm{~nm}$ in diameter were mixed with PEG-552 solution and placed at the water-octanol interface as previously described. Two different batches of the aforementioned mixture were analysed to investigate the batch-to-batch variation. Fig. S6a shows representative images of silica particles adsorbed at a water-octanol interface of different sizes. The computed contact angles with relative standard deviations are presented as a function of the size of the particles in Fig. S6b. The clear linear dependence of $\theta$ on the particle diameter is observed for both batches (red and purple data points) within the experimental error, providing experimental robustness to the observed linear trend.

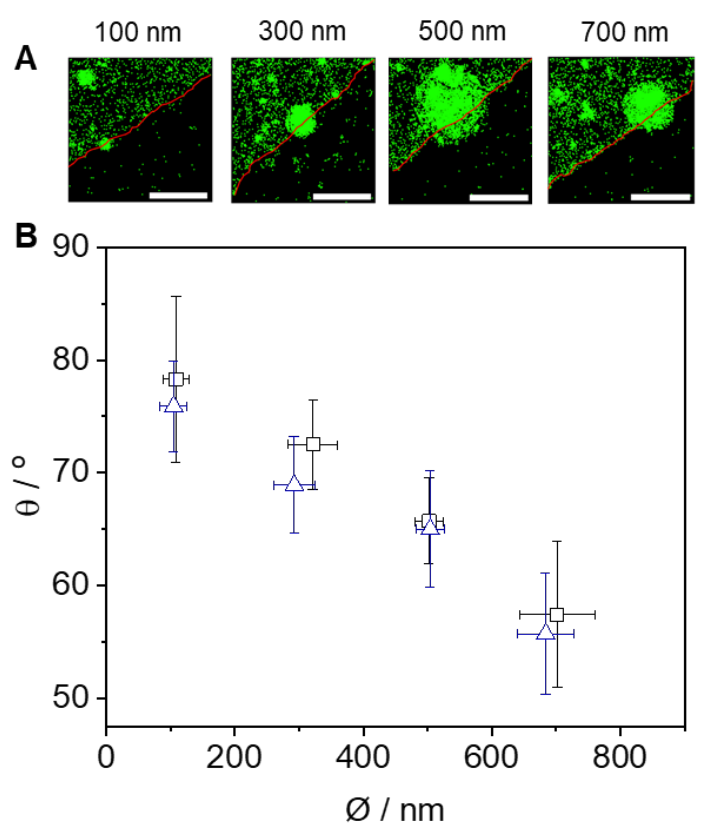

Fig. S6: (a) iPAINT images of single silica particles adsorbed at a water-octanol interface. The red line localizes the liquid-liquid interface between the aqueous phase (area filled with single molecule localizations - green dots) and the oil phase (black area). Scale bars $500 \mathrm{~nm}$. (b) Dependence of contact angle on particle size is shown for two different sets of silica particles black and navy data points). The contact angles have been calculated for $n>30$ particles for both batches. The error bars stem for the standard deviation on $\theta$ and the diameter. 


\section{Extended contact angle data.}

\section{1 $\theta$ vs. diameter for carboxyl-polystyrene and silica particles.}

The full data range of contact angles computed for carboxyl polystyrene latex (Fig. S7a) and silica (Fig. S7b) particles, respectively, are shown when adsorbed at water-octanol interfaces.
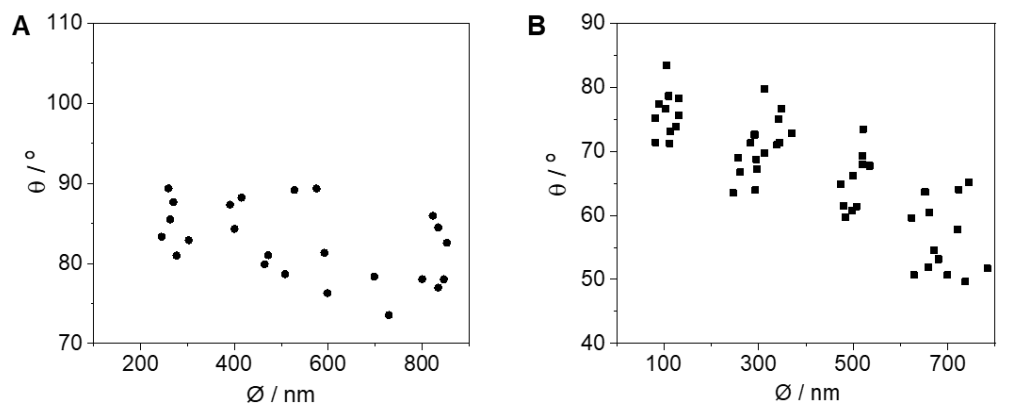

Fig. S7: Extended data of contact angle $\theta$ as function of particle diameter for (a) carboxyl polystyrene latex $(\mathrm{pH}=5)$, and $(\mathrm{b})$ silica colloids.

\section{2 $\theta$ vs. diameter for carboxyl-polystyrene particles at different $\mathrm{pH}$.}

The full data range of contact angles computed for carboxyl polystyrene latex particles are shown for $\mathrm{pH}=1.8$ (Fig. S8a), $\mathrm{pH}=4$ (Fig. S8b) and pH = 6 (Fig. S8c), respectively, when adsorbed at water-octanol interfaces.
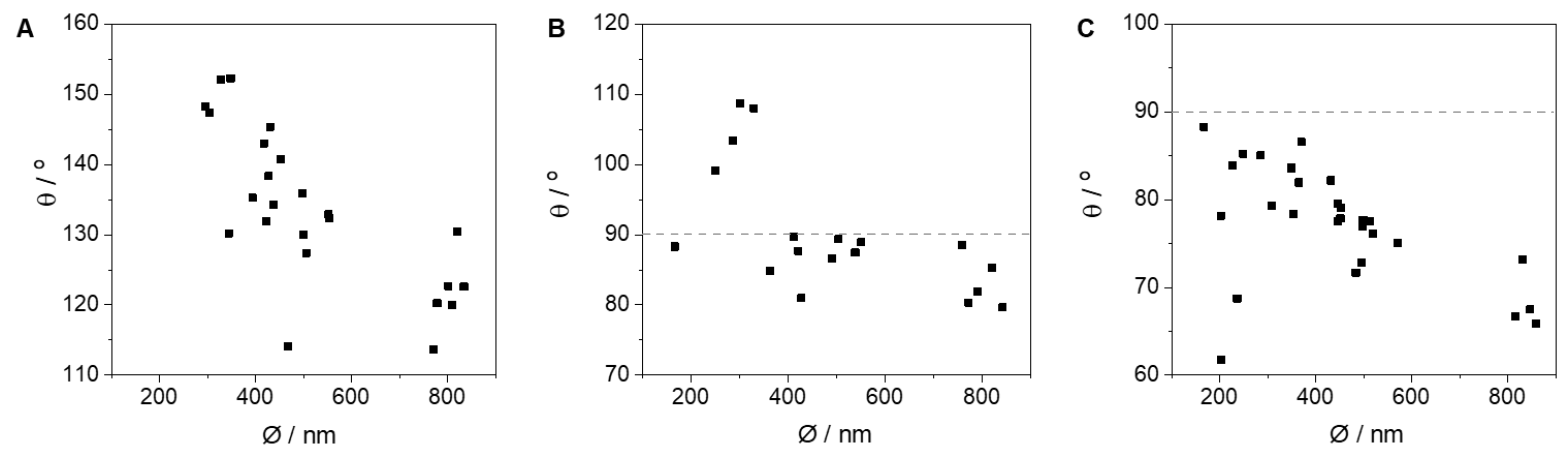

Fig. S8: Extended data of contact angle $\theta$ as function of particle diameter for carboxyl polystyrene latex particles at (a) $\mathrm{pH}=$ $1.8,(\mathrm{~b}) \mathrm{pH}=4$, and (c) $\mathrm{pH}=6$. 


\section{Contact angle dependency on pH.}

A complementary figure to Fig. 2 of the main text outlining the pH induced wettability shift for $320 \mathrm{~nm}$ CPS particles is presented as Fig. $\mathbf{S 9}$.
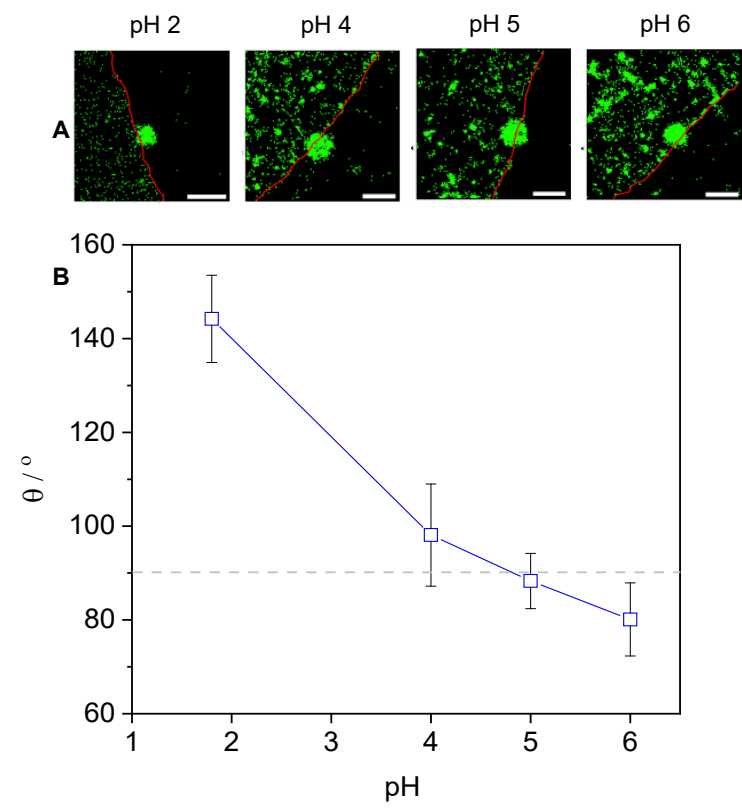

Fig. S9: Contact angles of $320 \mathrm{~nm}$ carboxyl polystyrene latex particles adsorbed the water-octanol interface at increasing $\mathrm{pH}$ values. The contact angles have been calculated for $n>30$ particles for each diameter. The error bars stem for the standard deviation on $\theta$.

The data shown in Fig. 2 of the main text is presented as $\theta$ vs. pH in full as Fig. S10, to highlight the effect of the $\mathrm{pH}$ on the contact angle. The contact angle of carboxyl polystyrene latex particles of different sizes $(320 \mathrm{~nm}, 450 \mathrm{~nm}, 510 \mathrm{~nm}$ and $810 \mathrm{~nm}$ ) at fluid interfaces have been plotted as a function of the $\mathrm{pH}$ of the particle solution.

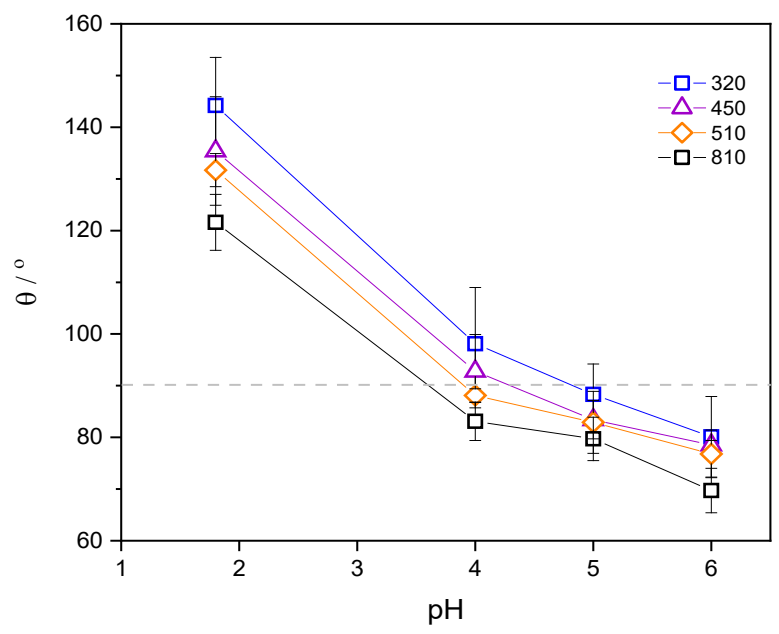

Fig. S10: Contact angles of carboxyl polystyrene latex particles with different diameters $(320 \mathrm{~nm}, 450 \mathrm{~nm}, 510 \mathrm{~nm}$ and 810 $\mathrm{nm}$ ) adsorbed at the water-octanol interface at increasing $\mathrm{pH}$ values. The contact angles have been calculated for $n>30$ particles for each diameter. The error bars stem for the standard deviation on $\theta$. 


\section{References.}

1. A. Aloi, N. Vilanova, L. Isa, A.M. de Jong, I.K. Voets, Nanoscale 2019, 11, 6654-6661. 